\title{
Tag der freien Berufe
}

Gabriela Rüttimann

Der «Tag der freien Berufe», durchgeführt am 10. Mai 2019, stand unter dem Eindruck der Auswirkungen der Digitalisierung auf Arbeitsplätze freier Berufe. Der Schweizer Verband freier Berufe führte diesen Anlass zum vierten Mal in Bern durch. Anlässlich unseres 40-jährigen Jubiläums war Gabriela Rüttimann als eine der Key-Speakerinnen eingeladen, um Digitalisierung aus der Perspektive der Psychotherapie zu thematisieren. Ihr Referat geben wir hier ungekürzt wieder.

\section{Wir brauchen endlich ein modernes Datenschutzgesetz}

Die Schweiz wartet seit Jahren auf ein neues Datenschutzgesetz, das den organisch gewachsenen Flickenteppich des jetzigen Gesetzes ablöst und die grossen Fragen des informationstechnologischen Zeitalters beantwortet.

Eine der grössten Herausforderungen unserer Zeit ist auch für die Psychotherapie die Digitalisierung und damit verbunden der Datenschutz. Die vielfältigen Online-Anwendungsmöglichkeiten sind Segen und Fluch zugleich. Für uns stellen sich verschiedene Fragen, die je nach Perspektive anders angegangen werden müssen und für die wir dringend stringente gesetzliche Leitlinien brauchen.

\section{Praktizierende Psychotherapeut*innen}

Aus Sicht der praktizierenden Psychotherapeut*innen müssen Berufsgeheimnis und Datensicherheit an erster Stelle stehen. Das muss im neuen Datenschutzgesetz (DSG) sichergestellt werden.

Da viele unserer Mitglieder und wohl auch Mitglieder anderer Berufskategorien keine «digital natives» sind, braucht es neben der Einführung des DSG auch Angebote zur Befähigung im Umgang mit der Digitalisierung. Wir stellen nämlich immer wieder fest, dass vielen unserer Mitglieder die Risiken im Umgang mit Online-Medien nicht bewusst sind. Das DSG muss in einer verständlichen Sprache vermittelt und plausibilisiert werden. Hier setzen wir uns für Fortbildungsangebote ein. Es braucht gesetzlich solide Anleitungen für Cloud-Lösungen und für den Datenaustausch sowie Lösungen, die in der Praxis umgesetzt werden können. Was geschieht zum Beispiel im Todesfall von Psychotherapeut*innen mit deren Akten? Oder was sind die
Auflagen bei der Aufgabe einer Psychotherapiepraxis?

Noch ist nicht klar, wohin die Reise mit dem Elektronischen Patient*innendossier (EPD) gehen wird. Im EPD sollen medizinische Berichte auf einer Plattform publiziert werden können. Wenn aber in solchen Datensätzen Fehler korrigiert werden müssen, verlangt der Datenschutz, dass alle, die einen entsprechenden Datensatz eingesehen oder bearbeitet haben, über etwaige Änderungen informiert werden. Das ist weder praktikabel noch sinnvoll. Das EPD sollte doch die Arbeit erleichtern und nicht erschweren. Im nächsten Jahr soll ein Monitoring zum EPD-Gesetz gestartet werden, was darauf hinweist, dass es noch dauern wird, bis das EPD grossräumig eingesetzt werden kann. Das EPD betrifft übrigens nicht nur unseren Beruf, sondern auch andere medizinische Berufe, die im SVFB zusammengeschlossen sind.

\section{Patient*innen}

Für Patient*innen steht der Schutz ihrer Privatsphäre an oberster Stelle. Sie müssen die Gewissheit haben, dass ihre Therapiebehandlungen absolut vertraulich bleiben. Psychische Probleme sind in unserer Gesellschaft nach wie vor stark stigmatisiert und können sich zum Beispiel auf die Arbeitsplatzsicherheit oder das soziale Umfeld negativ auswirken. Dieser geschützte Raum muss in jedem Fall beibehalten werden. Eine Patient*innendokumentation darf deshalb nur mit strikter Einwilligung respektive auf Wunsch der Patientin oder des Patienten an Dritte weitergegeben werden. Psychotherapie basiert stark auf gegenseitigem Vertrauen. Wird eine Psychotherapie online angeboten, müssen Patient*innen deshalb auf die absolute Diskretion der Therapierenden zählen können.

\section{Studierende}

Die Weiterbildung von Studierenden, die sich für eine Psychotherapieweiterbildung entschieden haben, muss das Thema Digitalisierung im Gesundheitsbereich beinhalten. Auch Jugendliche, die mit Online-Medien bestens vertraut sind, müssen für unsere Anliegen sensibilisiert werden. So fragte mich neulich eine Psychologiestudentin, was denn Big Data mit Psychotherapie zu tun habe. Ihr war wohl nicht bewusst, dass mit Big Data unter anderem auch grosser Unfug betrieben wird, was bei vielen Menschen

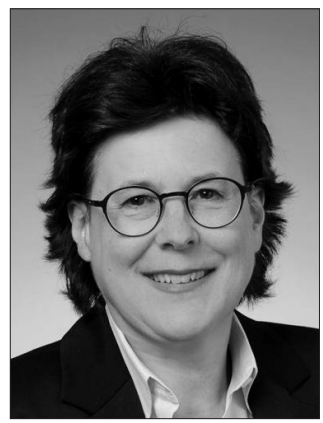


Unsicherheit und Ängste bis hin zu psychischen Störungen zur Folge hat. Sie hatte das Thema auf der Titelseite unseres Magazins à jour! entdeckt. Unser Anliegen ist deshalb, dass bei der nächsten Akkreditierungsrunde der Weiterbildungsinstitutionen, bei der sie ihre Curricula re-akkreditieren lassen müssen, das Thema Digitalisierung einbezogen wird.

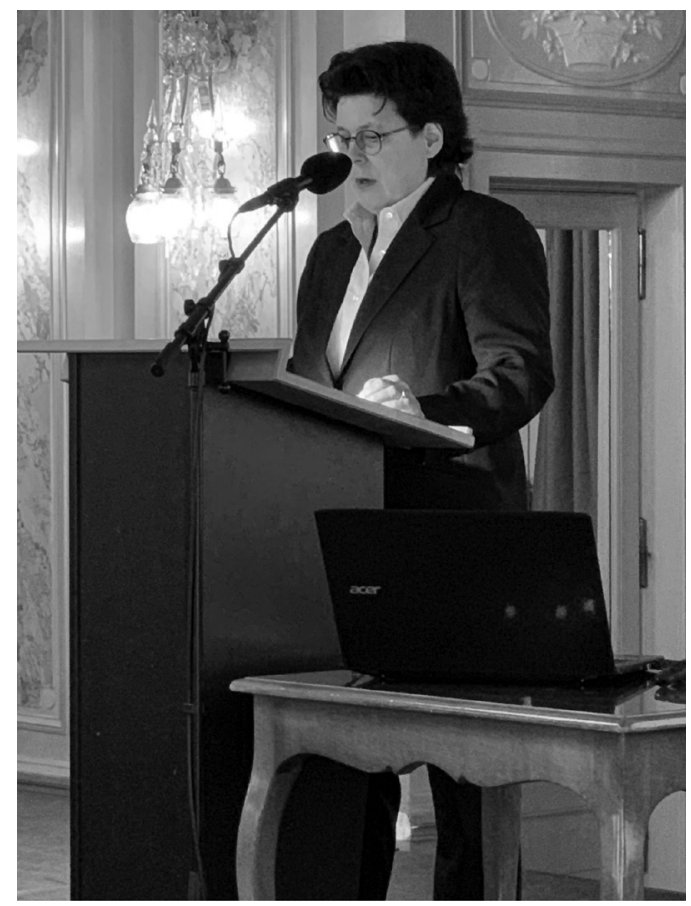

Gabriela Rüttimann am «Tag der freien Berufe»

\section{Berufsverband}

Für uns als Berufsverband der Psychotherapeutinnen und Psychotherapeuten gilt es im Bereich der Digitalisierung, die Anwendung computergestützter Psychotherapie, so genanntes Cognitive Computing, aufmerksam zu beobachten. Es sind bereits verschiedene Anwendungen auf dem Markt, und es ist unsere Aufgabe, die Spreu vom Weizen zu trennen und unseren Mitgliedern beratend zur Seite zu stehen. Die Erforschung solcher Anwendungen ist noch in den Anfängen und hat bislang kaum taugliche Anwendungsempfehlungen hervorgebracht. Wir beobachten die Entwicklung und sind mit verschiedenen Universitäten auf Tuchfühlung. IT-basierte Datenmengen bieten neue Möglichkeiten zur Optimierung der Leistungsfähigkeit der Medizin. Sie bergen aber auch Risiken. Dazu nur die Stichworte: Personalisierte Medizin, optimierte Diagnosestellung und Behandlungsassistenz anhand von Leitlinien. Natürlich haben an der Nutzung dieser Daten auch die Kostenträger ihr Interesse zur Senkung der Gesundheitskosten oder zur Risikoselektion der Versichernden. Im Bereich der Psychiatrie und Psychotherapie können Datenauswertungen ein weites Betätigungsfeld eröffnen. Bald kennen uns Maschinen besser als viele Menschen aus unserem Umfeld. Virtual Engineering ermöglicht zudem die Bearbeitung von Menschen in ihrem Denken, Fühlen und Handeln.

Für den Gesetzgeber wird es eine Mammutaufgabe sein, der rasch fortschreitenden Entwicklung zu folgen und uns ein Datenschutzgesetz an die Hand zu geben, das auch für Laien verständlich und anwendbar sein wird.

Unsere Aufgabe als Berufsverband wird es sein, ethische Leitlinien zu erarbeiten, um sicherzustellen, dass Computertechnologien auch wirklich zum Vorteil und nicht zum Nachteil einer höchst vulnerablen Zielgruppe, nämlich Menschen mit psychischen Problemen, eingesetzt werden.

Für all die aufgeworfenen Fragen brauchen wir endlich ein modernes Datenschutzgesetz.

Gabriela Rüttimann ist Präsidentin der ASP. 